\title{
LOWER BOUNDS ON THE NORMS OF EXTENSION OPERATORS FOR LIPSCHITZ DOMAINS
}

\author{
VLADIMIR LOTOREICHIK
}

Abstract. Let $\Omega \subset \mathbb{R}^{d}$ be a bounded or an unbounded Lipschitz domain. In this note we address the problem of continuation of functions from the Sobolev space $H^{1}(\Omega)$ up to functions in the Sobolev space $H^{1}\left(\mathbb{R}^{d}\right)$ via a linear operator. The minimal possible norm of such an operator is estimated from below in terms of spectral properties of self-adjoint Robin Laplacians on domains $\Omega$ and $\mathbb{R}^{d} \backslash \bar{\Omega}$. Another estimate of this norm is also given, where spectral properties of Schrödinger operators with the $\delta$-interaction supported on the hypersurface $\partial \Omega$ are involved. General results are illustrated with examples.

Mathematics subject classification (2010): 47A30, 46E35, 35P15, 47B38.

Keywords and phrases: Extension operator, Lipschitz domain, Robin Laplacian, Schrödinger operator with $\delta$-potential, norm estimates.

\section{REFERENCES}

[1] W. ARENDT AND R. MAZZEO, Friedlander's eigenvalue inequalities and the Dirichlet-to-Neumann semigroup, Commun. Pure Appl. Anal. 11 (2012), 2201-2212.

[2] W. ARENDT AND M. WARMA, The Laplacian with Robin boundary conditions on arbitrary domains, Potential Anal. 19 (2003), 341-363.

[3] J. BEHRNDT, P. EXNER AND V. LOTOREICHIK, Schrödinger operators with $\delta$ - and $\delta^{\prime}$-interactions on Lipschitz surfaces and chromatic numbers of associated partitions, arXiv:1307.0074.

[4] J. Behrndt, M. LAnger And V. LotoreICHIK, Schrödinger operators with $\delta$ - and $\delta^{\prime}$-potentials supported on hypersurfaces, Ann. Henri Poincaré 14 (2013), 385-423.

[5] M. Sh. Birman And M. Z. Solomjak, Spectral Theory of Selfadjoint Operators in Hilbert Spaces, Dordrecht, Holland, 1987.

[6] J. F. BRASChE, P. EXNER, YU. A. KUPERIN AND P. ŠEBA, Schrödinger operators with singular interactions, J. Math. Anal. Appl. 184 (1994), 112-139.

[7] V. I. BURENKOV, Extension theory for Sobolev spaces on open sets with Lipschitz boundaries, in Nonlinear analysis, function spaces and applications, Vol. 6 (1999), 1-49.

[8] A. P. CALderón, Lebesgue spaces of differentiable functions and distributions, Proc. Sympos. Pure Math. 4, (1961), 33-49.

[9] M. CostaBEL, Boundary integral operators on Lipschitz domains: elementary results, SIAM J. Math. Anal. 19 (1988), 613-626.

[10] Z. Ding, A proof of the trace theorem of Sobolev spaces on Lipschitz domains, Proc. Amer. Math. Soc. 124 (1996), 591-600.

[11] D. Edmunds And W. Evans, Spectral Theory and Differential Operators, The Clarendon Press, New York, 1987.

[12] P. EXNER, Leaky quantum graphs: a review, in: Analysis on Graphs and its Applications. Selected papers based on the Isaac Newton Institute for Mathematical Sciences programme, Cambridge, UK, 2007.

[13] F. Gesztesy And M. Mitrea, Nonlocal Robin Laplacians and some remarks on a paper by Filonov on eigenvalue inequalities, J. Differential Equations 247 (2009), 2871-2896.

[14] G. A. Kalyabin, Two-sided estimates for the least norm of extension operators from plane convex domains for Sobolev spaces, Proc. Steklov Inst. Math. 4 (1999), 140-145. 
[15] T. Kato, Perturbation Theory for Linear Operators, Reprint of the 1980 Edition, Springer-Verlag, Berlin, 1995.

[16] M. LeVitin And L. PARnovski, On the principal eigenvalue of a Robin problem with a large parameter, Math. Nachr. 281 (2008), 272-281.

[17] V. LotoReIchiK, Note on 2D Schrödinger operators with $\delta$-interactions on angles and crossing lines, Nanosystems: Phys. Chem. Math. 4 (2013), 166-172.

[18] W. MCLean, Strongly Elliptic Systems and Boundary Integral Equations, Cambridge University Press, Cambridge, 2000.

[19] J. MARSChalL, The trace of Sobolev-Slobodeckij spaces on Lipschitz domains, Manuscripta Math. 58 (1987), 47-65.

[20] V. MAZ'YA AND S. V. РoborChi, Extension of functions in Sobolev spaces on parameter dependent domains, Math. Nachr. 178 (1996), 5-41.

[21] S. G. Mikhlin, Equivalent norms in Sobolev spaces and norms of extension operators, Sibirsk. Mat. Zh. (1978), 1141-1153.

[22] S. G. MikHLIN, On the minimal extension constant for functions of Sobolev classes, Zap. Nauchn. Sem. Leningrad. Otdel. Mat. Inst. Steklov. (LOMI) 90 (1979), 150-185.

[23] S. G. MikHLin, Konstanten in einigen Ungleichungen der Analysis, Leipzig, Teubner, 1981.

[24] R. Seeley, Extension of $C^{\infty}$ functions defined in a half space, Proc. Amer. Math. Soc. 15 (1964), 625-626.

[25] E. M. Stein, Singular Integrals and Differentiability Properties of Functions, Princeton University Press, 1971. 